\title{
Comparisons between MHD model calculations and observations of Cassini flybys of Titan
}

\author{
Yingjuan Ma, ${ }^{1}$ Andrew F. Nagy, ${ }^{1}$ Thomas E. Cravens, ${ }^{2}$ Igor V. Sokolov, ${ }^{1}$ \\ Kenneth C. Hansen, ${ }^{1}$ Jan-Erik Wahlund, ${ }^{3}$ Frank J. Crary, ${ }^{4}$ Andrew J. Coates, ${ }^{5}$ \\ and Michele K. Dougherty ${ }^{6}$ \\ Received 18 October 2005; revised 25 January 2006; accepted 7 February 2006; published 20 May 2006.
}

[1] The Cassini spacecraft passed by Titan on 26 October 2004 (Ta flyby) and 13

December 2004 (Tb flyby). In both cases the Cassini Orbiter entered Titan's ionosphere and flew through Titan's dynamic wake region. In this paper, we present our simulation results of these two flybys using our three-dimensional multispecies MHD model. This model is a slightly updated version of the model outlined by Ma et al. (2004a); the main difference is the inclusion of magnetic diffusion into the equations. The calculations used the best available upstream plasma and magnetic field parameters obtained by the Cassini instrument complement. The calculated parameters, corresponding to the Cassini flybys near the closest approach, are compared with the relevant observed values. There is a reasonably good but clearly not perfect agreement between the measured and calculated values. Some of these differences are believed to be due to the uncertainties and time variability associated with the upstream parameters and some differences must definitely be the result of the uncertainties in the parameters selected for the model, as well as the limitations associated with the MHD approximations.

Citation: Ma, Y., A. F. Nagy, T. E. Cravens, I. V. Sokolov, K. C. Hansen, J.-E. Wahlund, F. J. Crary, A. J. Coates, and M. K. Dougherty (2006), Comparisons between MHD model calculations and observations of Cassini flybys of Titan, J. Geophys. Res., 111, A05207, doi:10.1029/2005JA011481.

\section{Introduction}

[2] On 1 July 2004 the Cassini spacecraft was successfully placed into orbit around Saturn and began comprehensive, observational studies of the Saturnian system. In anticipation of this extensive new database numerous theoretical models have been developed. In this paper we report on our three-dimensional (3-D), multispecies, nonideal, MHD model of the interaction of Saturn's magnetosphere with Titan. Earlier, in a brief publication [Ma et al., 2004a], we presented a prediction of this interaction based on Voyager 1 parameters. Here we describe our model in some more detail, present results corresponding to the Ta and $\mathrm{Tb}$ flybys of Titan, and compare the model predictions with the observed parameters.

\section{Model Description}

[3] The interaction of plasma flows (solar wind or magnetospheric plasmas) with "nonmagnetic" solar system

\footnotetext{
${ }^{1}$ Space Physics Research Laboratory, University of Michigan, Ann Arbor, Michigan, USA.

${ }^{2}$ Department of Physics and Astronomy, University of Kansas, Lawrence, Kansas, USA.

${ }^{3}$ Swedish Institute of Space Physics, Uppsala, Sweden.

${ }^{4}$ Southwest Research Institute, San Antonio, Texas, USA.

${ }^{5}$ Mullard Space Laboratory, University College London, London, UK.

${ }^{6}$ Blackett Laboratory, Imperial College, London, UK.

Copyright 2006 by the American Geophysical Union. 0148-0227/06/2005JA011481
}

bodies (e.g., Venus, Titan) is very different from those that have strong magnetic fields, such as the Earth or Jupiter. The obstacle to the plasma flow is the ionosphere/ atmosphere system, not the intrinsic magnetic field. The use of gasdynamic and later MHD models to study the interaction of the solar wind with the Earth began about 3 decades ago [e.g., Spreiter et al., 1966]. Variations of these models have also been successfully applied to other magnetic planets, such as Jupiter [e.g., Ogino et al., 1998; Hansen, 2001]. Both single and multifluid MHD models have also been used to study the interaction of fast plasma flows with "nonmagnetic" planets such as Venus and Mars [e.g., Spreiter et al., 1970; Murawski and Steinolfson, 1996; Bauske et al., 1998; Harnett and Winglee, 2003; Ma et al., 2004b] and moons such as Titan and Europa [e.g., Ledvina and Cravens, 1998; Nagy et al., 2001; Ma et al., 2004a, Backes et al., 2005]. Semikinetic models have also been employed to study the solar wind interaction with Mars and the magnetospheric plasma with Titan [e.g., Brecht, 1997; Brecht et al., 2000; Modolo et al., 2005]. These semikinetic model calculations have been very useful in showing the lack of symmetry in the interaction processes. It has been argued that the use of semikinetic models is especially appropriate for Mars and Titan, where the ion gyroradius is of the same order as the planetary radius. However, it is important to note that the MHD description has been found to be successful even in such situations. The possible reason for this maybe the fact that, as shown for Mars by the semikinetic codes and observed by the Phobos- 2 wave 
Table 1. Species and Group of Species Used in Current Titan Model

\begin{tabular}{ccccc}
\hline & Name & Components & Mass, amu & Mass Range, amu \\
\hline 1 & $\mathrm{~L}^{+}$ & $\mathrm{H}^{+}, \mathrm{H}_{2}^{+}, \mathrm{H}_{3}^{+}$ & 1 & $1-3$ \\
2 & $\mathrm{M}^{+}$ & $\mathrm{CH}_{5}^{+}, \mathrm{N}^{+}, \mathrm{CH}_{4}^{+}, \mathrm{CH}_{3}^{+}, \mathrm{CH}_{2}^{+}, \mathrm{CH}^{+}, \mathrm{C}^{+}$ & 14 & $12-17$ \\
3 & $\mathrm{H}^{+}$ & $\mathrm{C}_{2} \mathrm{H}_{5}^{+}$ & 29 & 29 \\
4 & $\mathrm{H}^{+}$ & $\mathrm{HCNH}^{+}$ & 28 & 28 \\
5 & $\mathrm{MHC}^{+}$ & $\mathrm{C}_{3} \mathrm{H}^{+}, \mathrm{C}_{3} \mathrm{H}_{2}^{+}, \mathrm{C}_{3} \mathrm{H}_{3}^{+}, \mathrm{C}_{3} \mathrm{H}_{4}^{+}, \mathrm{C}_{3} \mathrm{H}_{5}^{+}, \mathrm{C}_{4} \mathrm{H}_{3}^{+}, \mathrm{C}_{4} \mathrm{H}_{5}^{+}, \ldots$ & 44 & $37-53$ \\
6 & $\mathrm{HHC}^{+}$ & $\mathrm{C}_{5} \mathrm{H}_{3}^{+}, \mathrm{C}_{5} \mathrm{H}_{5}^{+}, \mathrm{C}_{5} \mathrm{H}_{7}^{+}, \mathrm{C}_{5} \mathrm{H}_{9}^{+}, \mathrm{C}_{6} \mathrm{H}_{5}^{+}, \mathrm{C}_{6} \mathrm{H}_{7}^{+}, \mathrm{C}_{7} \mathrm{H}_{5}^{+}, \ldots$ & 70 & $63-89$ \\
7 & $\mathrm{HNI}^{+}$ & $\mathrm{C}_{3} \mathrm{H}_{2} \mathrm{~N}^{+}, \mathrm{C}_{5} \mathrm{H}_{5} \mathrm{~N}^{+}, \mathrm{C}_{3} \mathrm{HN}^{+}$ & 74 & $51-79$ \\
\hline
\end{tabular}

instrument [Grard et al., 1989] and the MGS magnetometer [e.g., Acuña et al., 1998; Cloutier et al., 1999], significant wave activity and turbulence are present, leading to a wide variety of wave particle interactions, which in turn act as pseudocollisions. Also, it should be remembered that the magnetic field piles up inside the bow shock, thus reducing the gyroradius.

[4] Our 3-D, multispecies, MHD model is based on the so-called BATS-R-US (Block Adaptive-Tree Solar-Wind Roe-Type Upwind Scheme) model [c.f. Powell et al., 1999], which is the latest version of the single-fluid MHD code and has been developed for space physics application at the University of Michigan. The BATS-R-US solution method is a highly scalable, massively parallel, blockadaptive mesh refinement (Block-AMR) algorithm that makes use of recent advances in high-resolution upwind numerical methods. The basic elements of the modified solver are (1) a cell-centered upwind finite-volume approach that solves the hydrodynamic and electromagnetic equations in a tightly coupled manner (rather than in separate steps); (2) a flux function based on a new multiwave $(8-14)$ approximate Riemann solver that accounts for the propagation of MHD waves in a way that is stable, accurate, and conservative; (3) limited linear reconstruction that provides second-order accuracy away from high-gradient regions and monotonic non-oscillatory solutions throughout the computational domain; (4) explicit multistage time stepping; (5) block-based solution-adaptive grid and data structure; (6) physics-based refinement/coarsening; and (7) a parallel implementation that yields extremely high computational performance on a variety of massively parallel architectures. The resulting parallel adaptive upwind solution algorithm is both robust and accurate across a wide range of values of plasma $\beta$ (the ratio of thermal and magnetic pressures). However, even with the aid of solution-adaptive grids, resolving three-dimensional flows requires massive computing power. In order to obtain good altitude resolution we recently introduced a spherical grid system [Ma et al., 2004a; Ma et al., 2004b]. In this system, each block is equally spaced with respect to the natural logarithm of the radial distance, $r$, and the other two spherical coordinates $\Theta$ and $\Phi$. As in any finite volume method, each cell in the spherical mesh is a control volume over which the MHD equations are solved. In our formulation, the nodes of the grid are distributed spherically (ln $\mathrm{r}$, $\Theta, \Phi)$, however all faces of each cell are planar, being defined by the adjacent nodes and the normal to the plane. Each cell is therefore a six-sided polyhedron. Because cell interfaces are planes, the MHD equations can be solved in a Cartesian formulation (Cartesian derivatives).
[5] A comprehensive, one-dimensional, chemical equilibrium model has recently been updated and published by Cravens et al. [2005]. Clearly, such a complex chemical scheme cannot be incorporated into our MHD model. We are using a seven "species" chemical scheme (outlined in Table 1), which does a good job of describing the major chemistry at Titan, as the results indicate later in this paper. The effective chemical reaction rates were "created" by appropriate combination of the relevant reactions. A number of the reaction rates are temperature dependent [cf. Schunk and Nagy, 2000] and we used the electron temperature values calculated by Gan et al. [1992].

[6] The model includes ion productions of three primary ion species $\left(\mathrm{L}^{+}, \mathrm{M}^{+}\right.$, and $\left.\mathrm{H}^{+}\right)$from both solar radiation and superthermal electrons, taken from the work of Cravens et al. [2005]. The ionization rates due to solar radiation are calculated for different solar zenith angles ranging from zero up to $112^{\circ}$. At higher altitudes, significant ionization persists in the model for SZA up to $110^{\circ}$ due to the spherical nature of Titan's ionosphere. Plots of these production rates showing this effect for a very similar, but not identical neutral atmosphere, are shown by Cravens et al. [2004]. Secondary ionization, due to photoelectrons created as a by-product of the photoionization, is also included and forms an important part of the total dayside ionization rate at lower altitudes. A two-stream electron transport method [Nagy and Banks, 1970; Gan et al., 1992] is used to determine the photoelectron fluxes although the energy deposition of the photoelectrons is primarily local.

[7] Another source of superthermal electrons is Saturn's magnetosphere [Crary et al., 2006] and impact ionization of neutrals by these electrons is an important ion production mechanism on the nightside of Titan. We adopt the ion production rates associated with this source from Cravens et al. [2005], independent of solar zenith angle. The incident superthermal electron distribution adopted (and used as a boundary condition at the ends of the field lines) was Maxwellian with a density of $\mathrm{n}_{\mathrm{e}, \mathrm{mag}}=0.1 \mathrm{~cm}^{-3}$ and a temperature of $\mathrm{T}_{\mathrm{e} \text {,mag }}=100 \mathrm{eV}$. This is representative of outer magnetosphere electron populations according to Cassini measurements [Crary et al., 2006]. The draping of the magnetic field lines through Titan's ionosphere was approximated with parabolae, although the results are not terribly sensitive to the details of the field topology. Cravens et al. [2005] demonstrated that including the effects of magnetospheric electron precipitation is essential for obtaining reasonable agreement between calculated and measured ionospheric electron densities on the nightside of Titan for Ta conditions. 
[8] In the current seven-species Titan version of the model, we solve the following set of normalized MHD equations:

$$
\frac{\partial \mathbf{W}}{\partial t}+(\nabla \cdot \mathbf{F})^{T}=\mathbf{Q}
$$

the state vector $\mathbf{W}$, and flux tensor $\mathbf{F}$, in equation (1) are

$$
\begin{aligned}
& \mathbf{W}=\left(\begin{array}{c}
\rho_{i=1}^{\rho_{i}} \\
\sum_{i} \rho_{i} \\
\mathbf{B} \\
\varepsilon
\end{array}\right)
\end{aligned}
$$

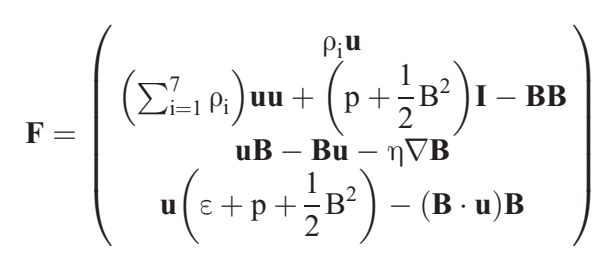

where $\varepsilon$ is the total energy density, defined as:

$$
\varepsilon=\frac{1}{2}\left(\sum_{\mathrm{i}=1}^{7} \rho_{\mathrm{i}}\right) \mathrm{u}^{2}+\frac{1}{\gamma-1} \mathrm{p}+\frac{\mathrm{B}^{2}}{2}
$$

The symbol $\rho_{\mathrm{i}}$, denotes the ion mass densities listed in Table 1, $\mathrm{p}$ is the total thermal pressure of the plasma, $\mathbf{u}$ is the velocity of the plasma. $\gamma$ is the ratio of specific heats (and taken to be 5/3). Here $\eta$ is the magnetic viscosity

$$
\eta=\frac{1}{\sigma_{0} \mu_{0}}
$$

where $\sigma_{0}$ is the electrical conductivity of the plasma. When the plasma is fully ionized, the electrical conductivity depends on the electron-ion collision frequency. In the lower ionosphere of Titan, especially on the nightside, the plasma is only partially ionized, where the electron-neutral collisions are more important. In the model the electrical conductivity is calculated using:

$$
\sigma_{0}=\frac{e^{2} n_{e}}{\sum_{n} \nu_{e n} m_{e}}
$$

Here, we included the collisions between the electrons and three major neutrals $\left(\mathrm{N}_{2}, \mathrm{CH}_{4}\right.$, and $\left.\mathrm{H}_{2}\right)$. The expressions for the collision frequencies $\left(v_{e n}\right)$ are taken from Keller [1992]. These collision frequencies are electron temperaturedependent, and we used $T_{p} / 2$ to estimate the value, where $\mathrm{T}_{\mathrm{p}}$ is the calculated plasma temperature. The collision frequencies are also proportional to the neutral densities. The magnetic diffusion term is only important in the nightside lower ionosphere region, where the neutral densities are high and electron densities are relatively low. At high altitude or in the dayside, the electrical conductivity is large, thus the nonideal effect is negligibly small.
[9] The source term in equation (1) is

$$
Q=\left(\begin{array}{c}
\left(\sum_{i=1}^{7} \rho_{i}\right) \mathbf{g}-\sum_{i=1}^{7}\left(\sum_{n=1}^{S_{i}} \rho_{i} L_{i} \nu_{i n}\right) \mathbf{u}-\mathbf{u}\left(\sum_{i=1}^{7} L_{i}\right) \\
\left(\sum_{i=1}^{7} \rho_{i}\right) \mathbf{u} \cdot \mathbf{g}-\frac{1}{2} \mathbf{u}^{2}\left(\sum_{i=1}^{7} L_{i}\right)-\frac{p}{\gamma-1}\left(\sum_{i=1}^{7} \frac{L_{i}}{m_{i}} / \sum_{i=1}^{7} \frac{\rho_{i}}{m_{i}}\right)+\frac{k T_{0}}{\gamma-1}\left(\sum_{i=1}^{7} \frac{S_{i}}{m_{i}}\right) \\
+\sum_{i=1}^{7}\left(\sum_{n=1}^{3} \frac{\rho_{i} v_{i n}}{m_{i}+m_{n}}\left[3 k\left(T_{n}-T_{i o n}\right)-m_{i} \mathbf{u}^{2}\right]\right)
\end{array}\right)
$$

where $\mathrm{S}_{\mathrm{i}}$ and $\mathrm{L}_{\mathrm{i}}$ denote the ion mass production and loss rates, respectively. The $\nu_{i n}$ is the momentum transfer collision frequency between ions and neutrals, which can be expressed as [Schunk and Nagy, 2000]:

$$
\nu_{i n}=2.21 \pi \frac{n_{n} m_{n}}{m_{i}+m_{n}}\left(\frac{\gamma_{n} e^{2}}{\mu_{i n}}\right)^{1 / 2}
$$

where $\mu_{i n}$ is the reduced mass and $\gamma_{n}$ is the neutral polarizability and

$$
\gamma_{n}=\alpha_{n} \times 10^{-24} \mathrm{~cm}^{3}
$$

The polarizability parameter $\alpha_{n}$ is $1.76,2.59$, and 0.82 for $\mathrm{N}_{2}, \mathrm{CH}_{4}$, and $\mathrm{H}_{2}$, respectively. In Equation (7), $\mathrm{T}_{\mathrm{n}}$ is the neutral temperature. It is taken to be $150 \mathrm{~K}$, to be consistent with the Cassini INMS observations [Waite et al., 2005]. $\mathrm{T}_{\mathrm{o}}$ is the temperature of the newly produced ions, which is assumed to be the same as $T_{n}$, the temperature of local neutral atmosphere.

[10] The reference frame used in the model is set as follows: the $\mathrm{X}$ axis is along the corotation direction, the positive $\mathrm{Y}$ direction is from Titan to Saturn, and the $\mathrm{Z}$ axis completes the right-handed coordinate system. In order to avoid any possible effects due to the assumed outer boundary conditions, we use a very large computational domain, given by $-16 \mathrm{R}_{\mathrm{T}} \leq \mathrm{X} \leq 48 \mathrm{R}_{\mathrm{T}},-32 \mathrm{R}_{\mathrm{T}} \leq \mathrm{Y}, \mathrm{Z} \leq 32 \mathrm{R}_{\mathrm{T}}$. In these Titan calculations the minimum radial grid size is about $36 \mathrm{~km}$. Our inner boundary was at $725 \mathrm{~km}$ above the surface and the ion densities were set at their chemical equilibrium values.

\section{Simulation Results and Comparisons With Observations}

3.1. Ta Flyby

[11] The first close flyby of Titan by Cassini took place on 26 October 2004 and is referred to as the Ta flyby. At closest approach (CA), which occurred at 1530 UT, the spacecraft came within $1174 \mathrm{~km}$ of Titan's surface. The flyby geometry is shown in Figure 1. At that time the subsolar location was at $70^{\circ} \mathrm{E}, 23^{\circ} \mathrm{S}$ in the reference frame indicated before and Titan was located at 10.6 hours Saturn local time (LT). The observational data from the Cassini Plasma Spectrometer (CAPS) [Young et al., 2004] and Cassini magnetometer (MAG) [Dougherty et al., 2004] show significant variability in the surrounding plasma environments as a function of time/location. CAPS data show that the inbound electron number density varied from $0.07 \mathrm{~cm}^{-3}$ to $1.92 \mathrm{~cm}^{-3}$ between $1300 \mathrm{UT}$ to $1500 \mathrm{UT}$, 


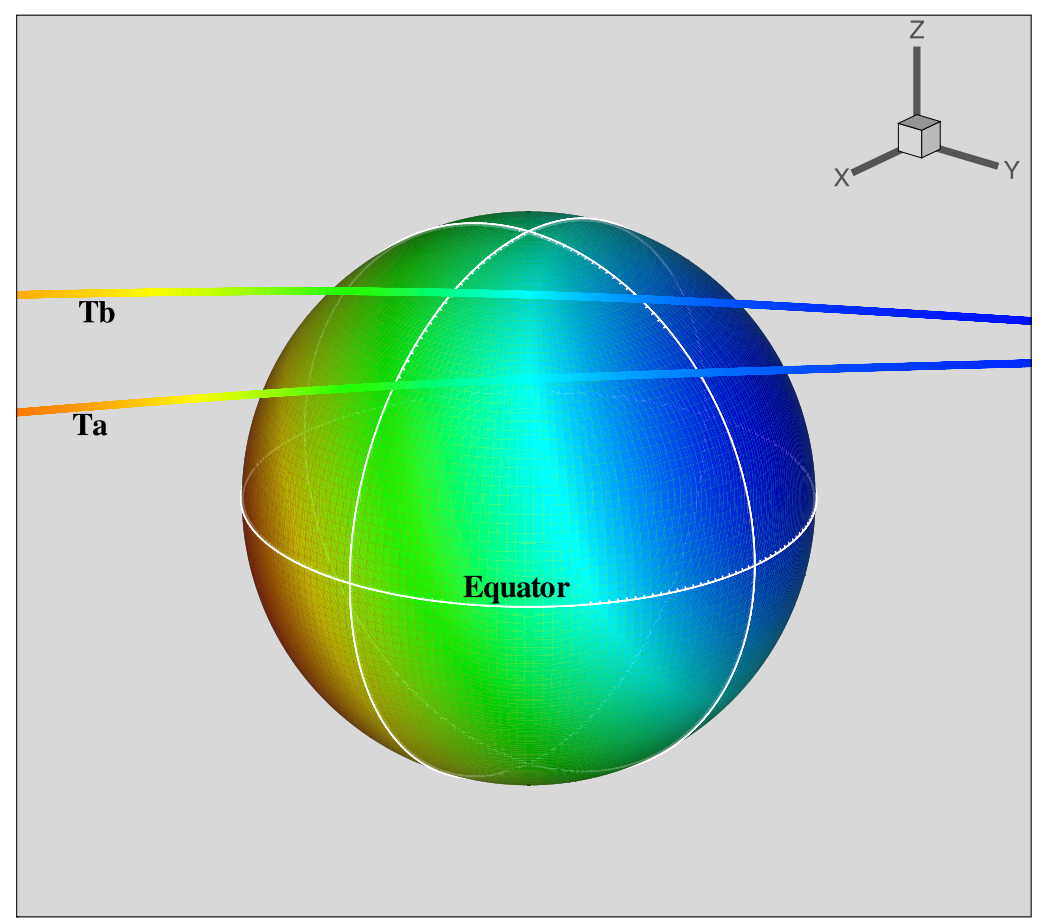

Figure 1. Sketch of the Ta and $\mathrm{Tb}$ flyby trajectory. The color plots show the $\cos (\mathrm{SZA})$, which indicates the orientation of the Sun.

giving an average value of about $0.41 \mathrm{~cm}^{-3}$. The electron temperature values ranged between $6.6 \mathrm{eV}$ and $97.2 \mathrm{eV}$ giving a mean value around $32.7 \mathrm{eV}$ (corresponding to $3.8 \times 10^{5} \mathrm{~K}$ ). CAPS data also show a significant difference between the inbound and outbound values in the electron density. The averaged outbound (from 1600 UT to 1800 UT) electron density decreased significantly to $0.23 \mathrm{~cm}^{-3}$. The electron temperature varied from $13.2 \mathrm{eV}$ to $98.7 \mathrm{eV}$, with the averaged electron temperature slightly larger than the inbound value. The CAPS ion data indicated an upstream speed between 120 and $160 \mathrm{~km} / \mathrm{s}$ according to Szego et al. [2005], but Crary et al. [2006] find the speed to be $110 \pm$ $20 \mathrm{~km} / \mathrm{s}$. The magnetic field data from MAG team show the averaged inbound magnetic field is very different from the mean outbound magnetic field (see Table 2).

[12] Given this variability, it is not easy to select for the model calculations an appropriate value for the upstream plasma parameters. The values we selected for our model calculations are listed in Table 3 . The upstream magnetic field was set as $\mathbf{B}=(1.25,3.0,-5.0) \mathrm{nT}$. The upstream electron number densities were set to be $0.3 \mathrm{~cm}^{-3}$. Both the magnetic field and electron density are chosen as the averages of the inbound and outbound values. We assume that the upstream plasma ions are composed of $75 \%$ of $\mathrm{L}^{+}$ ions and $25 \%$ of $\mathrm{M}^{+}$ions, corresponding to an average ion mass of $4.25 \mathrm{amu}$, consistent with the early Cassini observations [Crary et al., 2006]. On the basis of the limited information, the plasma temperature, $T_{p}$ is picked to be $350 \mathrm{eV}$ and the plasma velocity is taken to be $\mathrm{u}=$ $120 \mathrm{~km} / \mathrm{s}$, with a $20^{\circ}$ northward and $10^{\circ}$ anti-Saturn direction component. These parameters correspond to a supersonic $\left(\mathrm{M}_{\mathrm{S}}=1.05\right)$, super-Alfvenic $\left(\mathrm{M}_{\mathrm{A}}=1.04\right)$, subfast-magnetosonic $\left(\mathrm{M}_{\mathrm{f}}=0.96\right)$ flow, and a plasma $\beta=1.19$, slightly larger than one.
[13] Figure 2 shows the calculated velocities in the equatorial $(\mathrm{X}-\mathrm{Y})$ and vertical $(\mathrm{X}-\mathrm{Z})$ planes for Ta flyby. The color plots show the speed of the plasma and the black arrows show the direction of the flow vector in the corresponding planes. There is no significant difference in the flow patterns between the equatorial and vertical planes in the present case, but when Voyager like upstream conditions were used, $M a$ et al. [2004a] found different flow patterns in the two planes. The difference is caused by the presence of a significant $\mathrm{Y}$ component of the magnetic field for the current case. The plasma flow gradually slows down, starting around $10 \mathrm{R}_{\mathrm{T}}$ along the ram direction mainly due to the mass loading process. The flow speed increases noticeably in the flank regions as the plasma moves past Titan.

Table 2. Trajectory Parameters and Observational Data for Ta and Tb Flybys

\begin{tabular}{|c|c|c|}
\hline & $\mathrm{Ta}$ & $\mathrm{Tb}$ \\
\hline CA time & 26 Oct 2004,1530 & 13 Dec 2004,1138 \\
\hline $\mathrm{CA}$ altitude, $\mathrm{km}$ & 1174 & 1200 \\
\hline SLT & 10.6 & 10.5 \\
\hline $\operatorname{Max} \mathrm{n}_{\mathrm{e}}, \mathrm{cm}^{-3}$ & $3.9 \times 10^{3}$ & $3.2 \times 10^{3}$ \\
\hline $\mathrm{CA} \mathrm{n}_{\mathrm{e}}, \mathrm{cm}^{-3}$ & $2.55 \times 10^{3}$ & $1.04 \times 10^{3}$ \\
\hline $\mathrm{CA} \mathrm{T}_{\mathrm{e}}, \mathrm{K}$ & 1132.0 & 1142.4 \\
\hline Inbound & $1300-1500$ & $0900-1100$ \\
\hline $\mathrm{B}_{\mathrm{in}}, \mathrm{nT}$ & $(0.51,2.55,-5.80)$ & $(1.83,2.78,-4.27)$ \\
\hline$\left|\mathrm{B}_{\text {in }}\right|, \mathrm{nT}$ & 6.35 & 5.42 \\
\hline $\mathrm{n}_{\mathrm{e}}, \mathrm{cm}^{-3}$ & 0.4 & 0.06 \\
\hline $\mathrm{T}_{\mathrm{e}}, \mathrm{eV}$ & 32.7 & 64.7 \\
\hline Outbound & $1600-1800$ & $1210-1410$ \\
\hline $\mathrm{B}_{\text {out }}, \mathrm{nT}$ & $(2.00,3.47,-4.11)$ & $(0.60,2.27,-2.89)$ \\
\hline$\left|\mathrm{B}_{\text {out }}\right|, \mathrm{nT}$ & 5.74 & 3.72 \\
\hline $\mathrm{n}_{\mathrm{e}}, \mathrm{cm}^{-3}$ & 0.23 & 0.14 \\
\hline $\mathrm{T}_{\mathrm{e}}, \mathrm{eV}$ & 37.9 & 87.3 \\
\hline
\end{tabular}


Table 3. Incident Plasma Parameters Adopted in the Model Calculations (in TIIS Coordinate)

\begin{tabular}{|c|c|c|c|}
\hline & Voyager Condition & Cassini Condition, Ta & Cassini Condition, $\mathrm{Tb}$ \\
\hline Magnetic field B, nT & $(1.6,0.05,-4.99)|\mathrm{B}|=5.2 \mathrm{nT}$ & $(1.25,3.0,-5.0)|\mathrm{B}|=6.0 \mathrm{nT}$ & $(1.25,2.5,-3.5)|\mathrm{B}|=4.5 \mathrm{nT}$ \\
\hline Plasma flow speed $\mathrm{u}, \mathrm{km} / \mathrm{s}$ & $(113,41,0)|\mathrm{u}|=120 \mathrm{~km} / \mathrm{s}$ & $(111,-21,41)|\mathrm{u}|=120 \mathrm{~km} / \mathrm{s}$ & $(99,-63,43)|\mathrm{u}|=125 \mathrm{~km} / \mathrm{s}$ \\
\hline Electron number density $n_{e}$ & $0.3 \mathrm{~cm}^{-3}$ & $0.3 \mathrm{~cm}^{-3}$ & $0.1 \mathrm{~cm}^{-3}$ \\
\hline $\mathrm{L}^{+}$number density $n_{L}^{+}$ & $0.1 \mathrm{~cm}^{-3}$ & $0.225 \mathrm{~cm}^{-3}$ & $0.08 \mathrm{~cm}^{-3}$ \\
\hline $\mathrm{M}^{+}$number density $n_{M}^{+}$ & $0.2 \mathrm{~cm}^{-3}$ & $0.075 \mathrm{~cm}^{-3}$ & $0.02 \mathrm{~cm}^{-3}$ \\
\hline Plasma temperature $k T_{p}$ & $2200 \mathrm{eV}$ & $350 \mathrm{eV}$ & $750 \mathrm{eV}$ \\
\hline Mass density $\rho$ & $2.9 \mathrm{amu} / \mathrm{cm}^{3}$ & $1.275 \mathrm{amu} / \mathrm{cm}^{3}$ & $0.36 \mathrm{amu} / \mathrm{cm}^{3}$ \\
\hline Total plasma pressure $P$ & $1.06 \times 10^{-10} \mathrm{~N} / \mathrm{m}^{2}$ & $1.7 \times 10^{-11} \mathrm{~N} / \mathrm{m}^{2}$ & $1.2 \times 10^{-11} \mathrm{~N} / \mathrm{m}^{2}$ \\
\hline$\beta=\mathrm{P} /\left(\mathrm{B}^{2} / 2 \mu_{0}\right)$ & 9.69 & 1.19 & 1.50 \\
\hline Alfven speed $c_{A}$ & $67 \mathrm{~km} / \mathrm{s}$ & $115.2 \mathrm{~km} / \mathrm{s}$ & $162.8 \mathrm{~km} / \mathrm{s}$ \\
\hline Sonic speed $c_{S}$ & $190 \mathrm{~km} / \mathrm{s}$ & $114.6 \mathrm{~km} / \mathrm{s}$ & $182.3 \mathrm{~km} / \mathrm{s}$ \\
\hline Fast magnetosonic speed $c_{f}$ & $191 \mathrm{~km} / \mathrm{s}$ & $124.8 \mathrm{~km} / \mathrm{s}$ & $200.2 \mathrm{~km} / \mathrm{s}$ \\
\hline Alfven Mach number $M_{A}=u / c_{A}$ & 1.79 & 1.04 & 0.77 \\
\hline Sonic Mach number $M_{s}=u / c_{s}$ & 0.63 & 1.05 & 0.68 \\
\hline Fast magnetosonic Mach number $M_{f}=u / c_{f}$ & 0.63 & 0.96 & 0.62 \\
\hline
\end{tabular}

The velocity in the X-Y plane reaches about $182 \mathrm{~km} / \mathrm{s}$, which is about $52 \%$ larger than the upstream corotating flow speed. This acceleration is mainly driven by the magnetic tension force. Close to the body, the plasma flow clearly diverts around Titan, and the flow speed drops sharply to a value close to zero due to the mass loading process.

[14] The calculated magnetic fields in the X-Y and X-Z planes for Ta flyby are shown in Figure 3. The color plots represent the magnitude of the total field and the black arrows show the direction of the magnetic field in the corresponding planes. The field lines are highly draped in the region close to Titan. The pile up of the magnetic field in front of Titan, due to the slowing down of the plasma flow, is clearly visible. A small current sheet is present in the wake region, where the magnetic field strength is close to zero.

[15] The calculated major ions and electron densities and ion temperature (assumed to be given by $\mathrm{T}_{\mathrm{p}} / 2$ ), corresponding to a solar zenith angle (SZA) of $58.9^{\circ}$ are plotted in Figure 4, along with the chemical equilibrium model results provided by the Kansas group [Cravens et al., 2005]; note that the two different ram angle conditions correspond to the same SZA. The agreement between our seven-ion model and the Kansas 51 ion model is reasonably good below about $1300 \mathrm{~km}$. The significant difference between the results in the region above about $1300 \mathrm{~km}$ is an

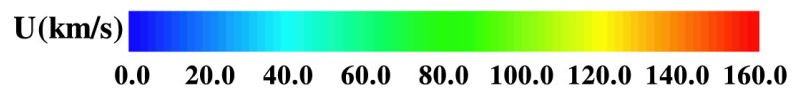

$\mathbf{U}(\mathbf{k m} / \mathbf{s})$
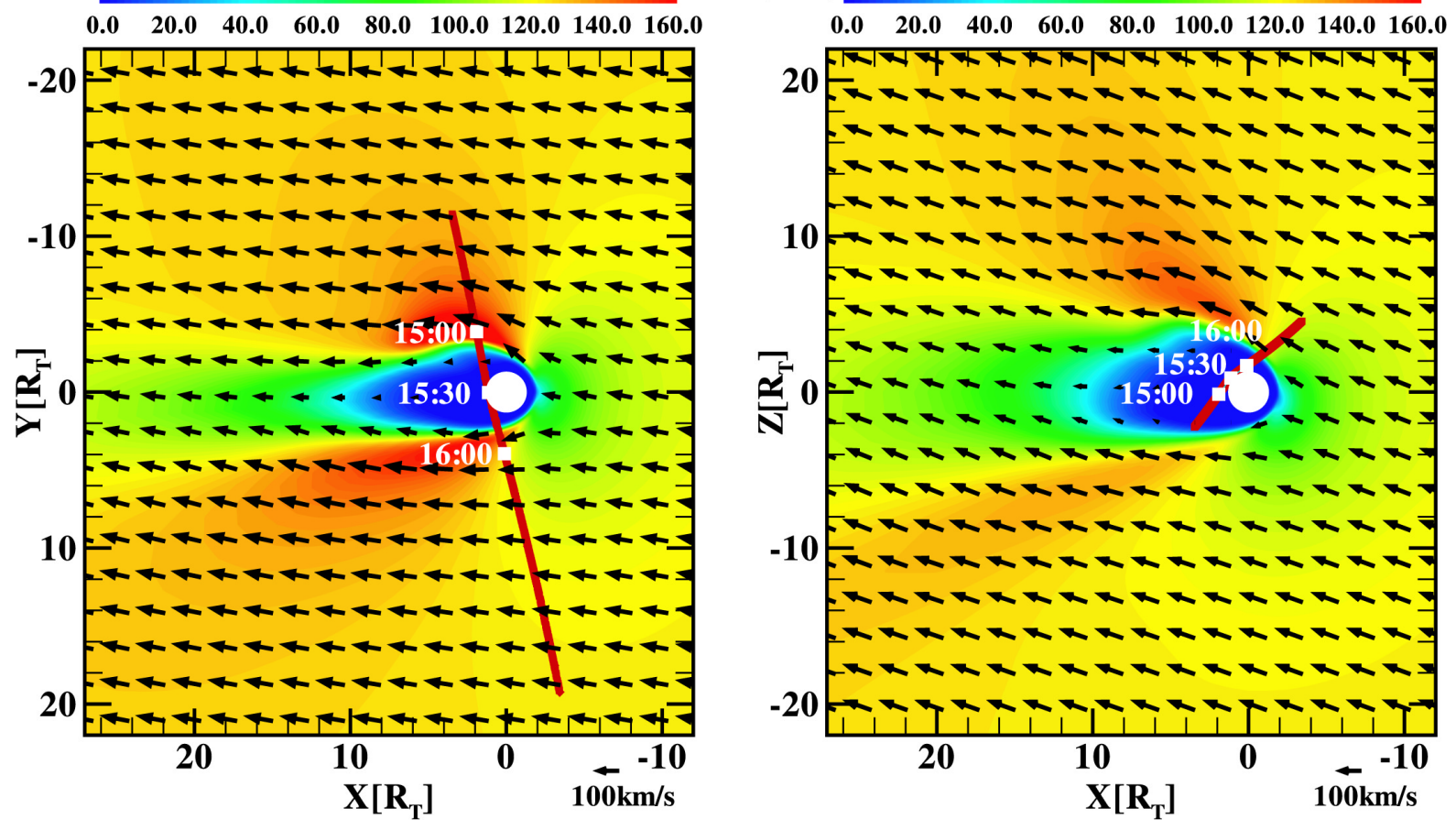

Figure 2. Calculated velocity values in $X-Y$ and $X-Z$ planes for Ta flyby. The color plots show the speed of the plasma, and the black arrows show the direction of the flow vector in the corresponding planes. 

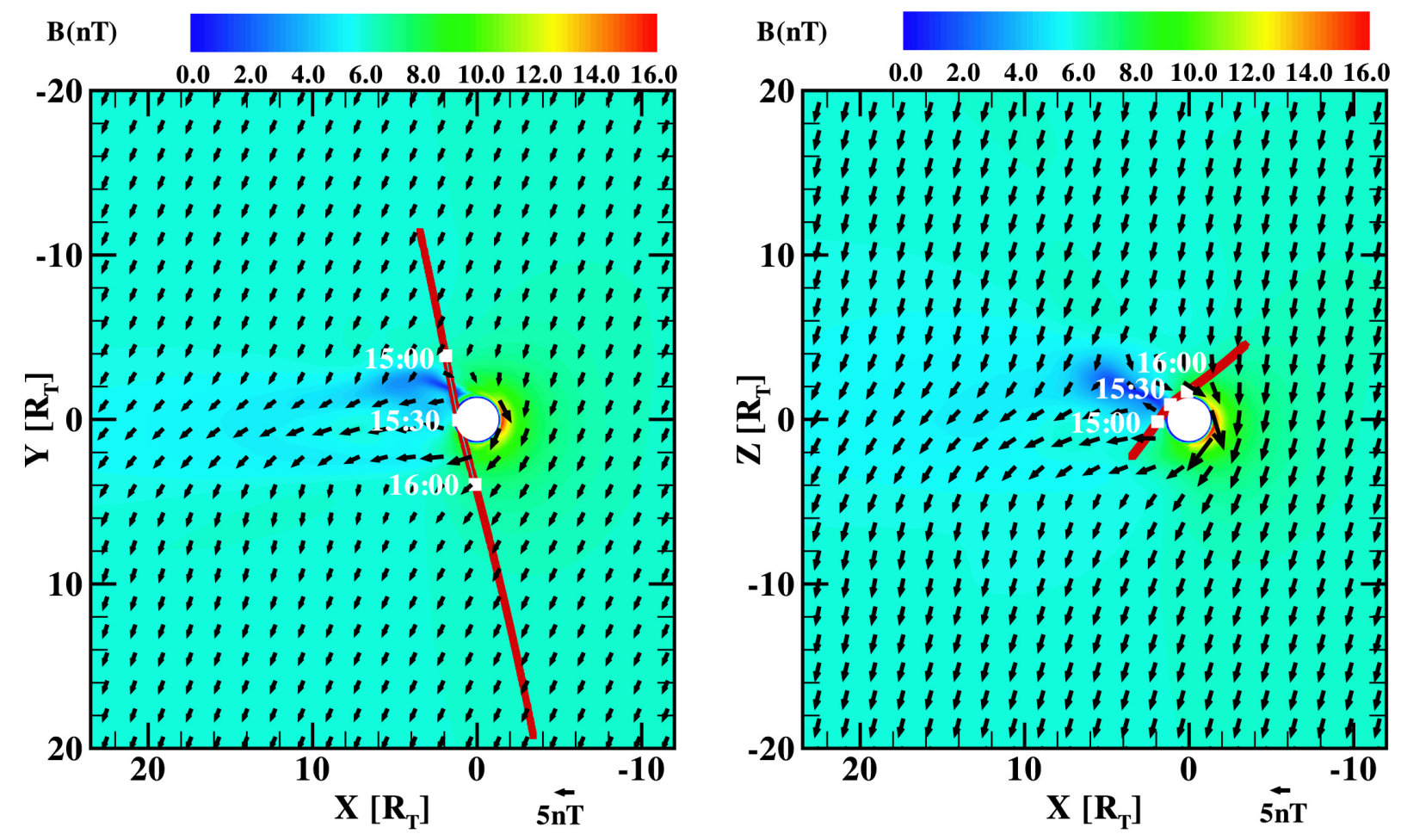

Figure 3. Calculated magnetic field values in $X-Y$ and $X-Z$ planes for Ta flyby. The color plots represent the strength of the magnetic field, and the black arrows indicate the direction of the magnetic field in the corresponding planes.

indication of the importance and effect of transport at these higher altitudes. The calculated densities for the different ram conditions are different at the higher altitudes, due to the different flow conditions, as one would expect.
The calculated plasma temperature is the same as the neutral temperature below about $1400 \mathrm{~km}$ due to the close collisional coupling between the ions and neutrals. When the collisions are less frequent at high altitudes, mainly due to
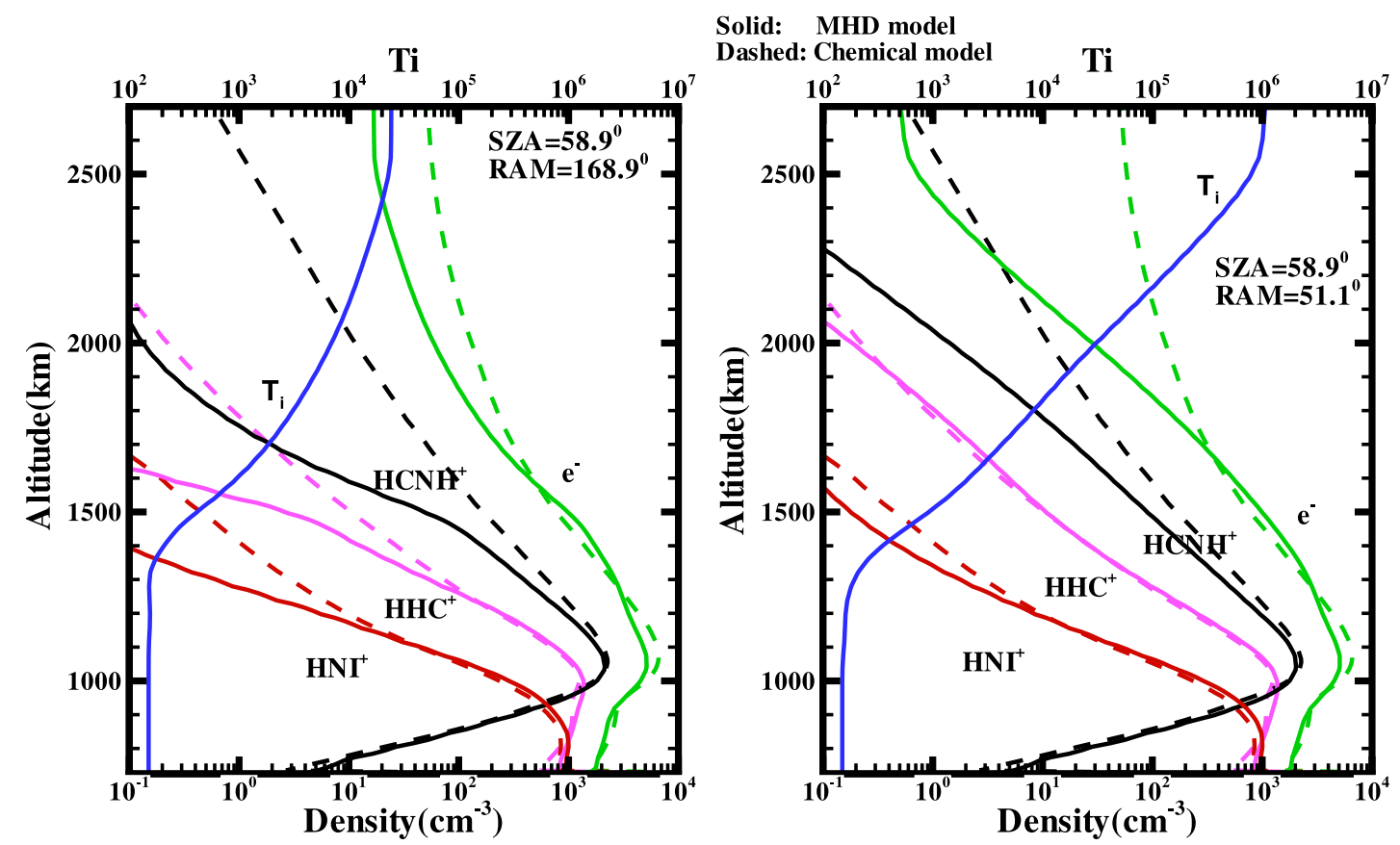

Figure 4. Densities calculated by the three-dimensional, MHD model along $60^{\circ}$ solar zenith angle, compared with the results from the Kansas, one-dimensional, comprehensive chemical equilibrium model. 

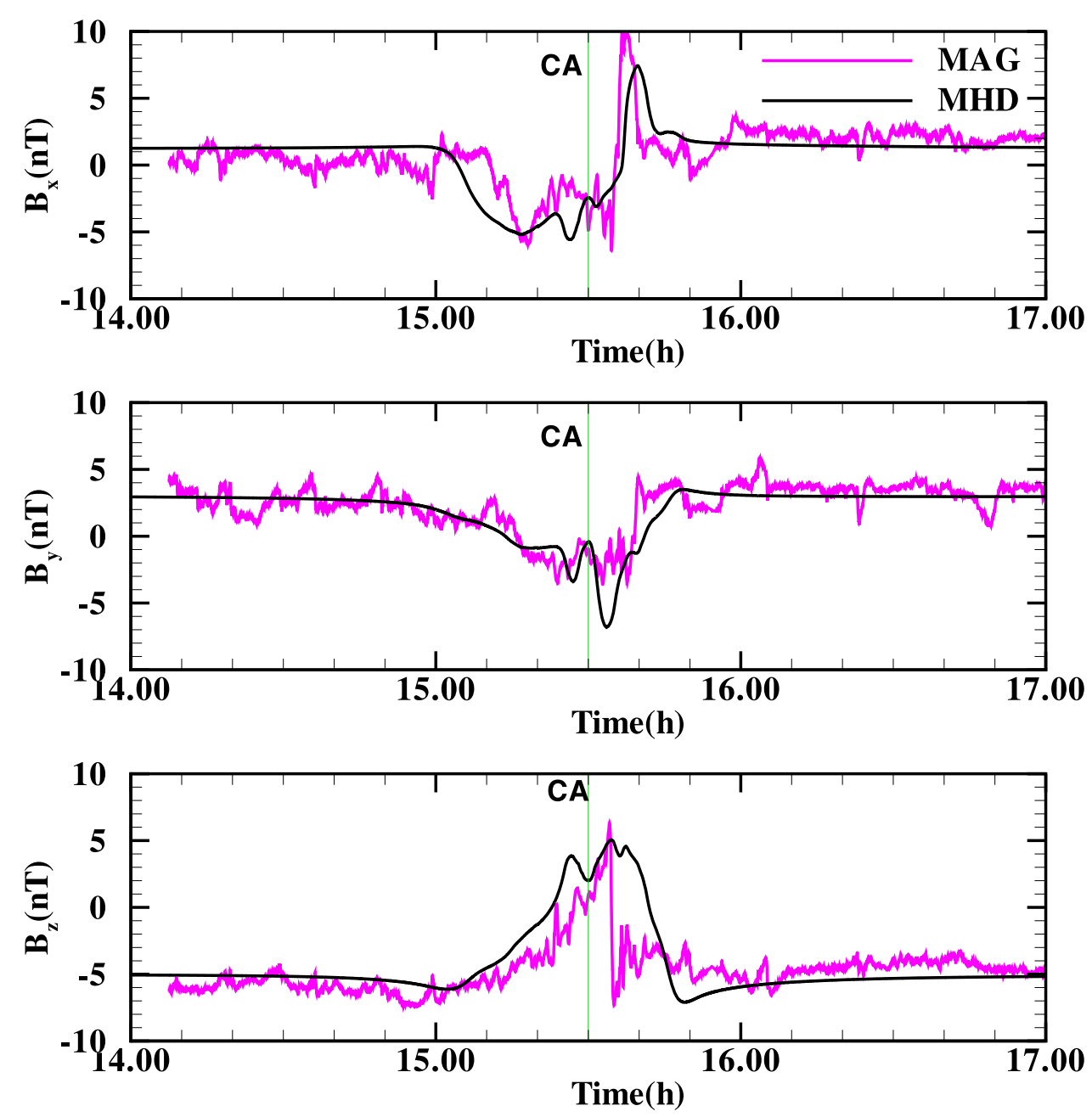

Figure 5. Calculated and measured magnetic field values along the Cassini Ta trajectory near closest approach. The green lines correspond to the closest approach time 1530 UT.

the decrease of neutral densities, the plasma temperature starts to increase sharply, especially on the ramside where the flow velocities are faster.

[16] Figure 5 shows the comparison between the calculated and observed magnetic field values along the Ta trajectory for plus and minus 1.5 hours of closest approach (CA), which is indicated by the green lines. The gross features of the observed, rapid changes in the $\mathrm{B}_{\mathrm{y}}$ and $\mathrm{B}_{\mathrm{z}}$ components near $\mathrm{CA}$ are reasonably well reproduced by the model. The observed peak after $\mathrm{CA}$ in the $\mathrm{B}_{\mathrm{x}}$ component is also present in the model.

[17] A comparison between the calculated and observed plasma parameters are shown in Figure 6, during the 1.5 hours interval centered on the closest approach (CA), which is indicated by the green lines. The top part of the figure shows a reasonably good agreement between the calculated electron density and the Langmuir probe [Wahlund et al., 2005] values in the ionospheric region. The plot clearly shows that within the ionosphere, where the electron energies are a fraction of an $\mathrm{eV}$, the Langmuir probe provides the correct information on the total electron densities, while outside this region, when the electron energies exceed $10 \mathrm{~s}$ of $\mathrm{eV}$, the CAPS electron detector is the instrument to rely on (see the transitions around 1520 and 1540). Both the simulation results and LP data show no large asymmetry in the mass-loading region between the inbound and outbound passes [Wahlund et al., 2005]. The electron densities from the Langmuir probe peaked $1.5 \mathrm{~min}$ before the CA with a maximum value of $3.9 \times 10^{3} \mathrm{~cm}^{-3}$ [Cravens et al., 2005]. The model predicted that the peak density is at about the same time, with a slightly smaller value $3.1 \times 10^{3} \mathrm{~cm}^{-3}$. The electron density discrepancy is likely to be due to the electron temperature used to calculate the recombination reaction rate. The electron temperature we adopted is from Gan et al. [1992], which gives $\mathrm{Te}=$ $600 \mathrm{~K}$ near $1200 \mathrm{~km}$, while the measured Te by the Langmuir probe is about $1300 \mathrm{~K}$. This difference in the electron temperature can affect the electron density by about $25 \%$ [Cravens et al., 2005]. The middle panel shows the calculated velocities along with those obtained by the Langmuir probe. Our model results show that Cassini would have crossed the deceleration region (from a velocity of $150 \mathrm{~km} / \mathrm{s}$ down to $10 \mathrm{~km} / \mathrm{s}$ ) in about 10 min during the inbound, while the observational data indicate longer intervals $(\sim 15 \mathrm{~min})$ for the Ta flyby [Szego et al., 2005]. This might be related to finite gyroradius effects, which are neglected in the MHD model. The bottom panel shows calculated and measured temperatures. Here again it is clearly shown that the 

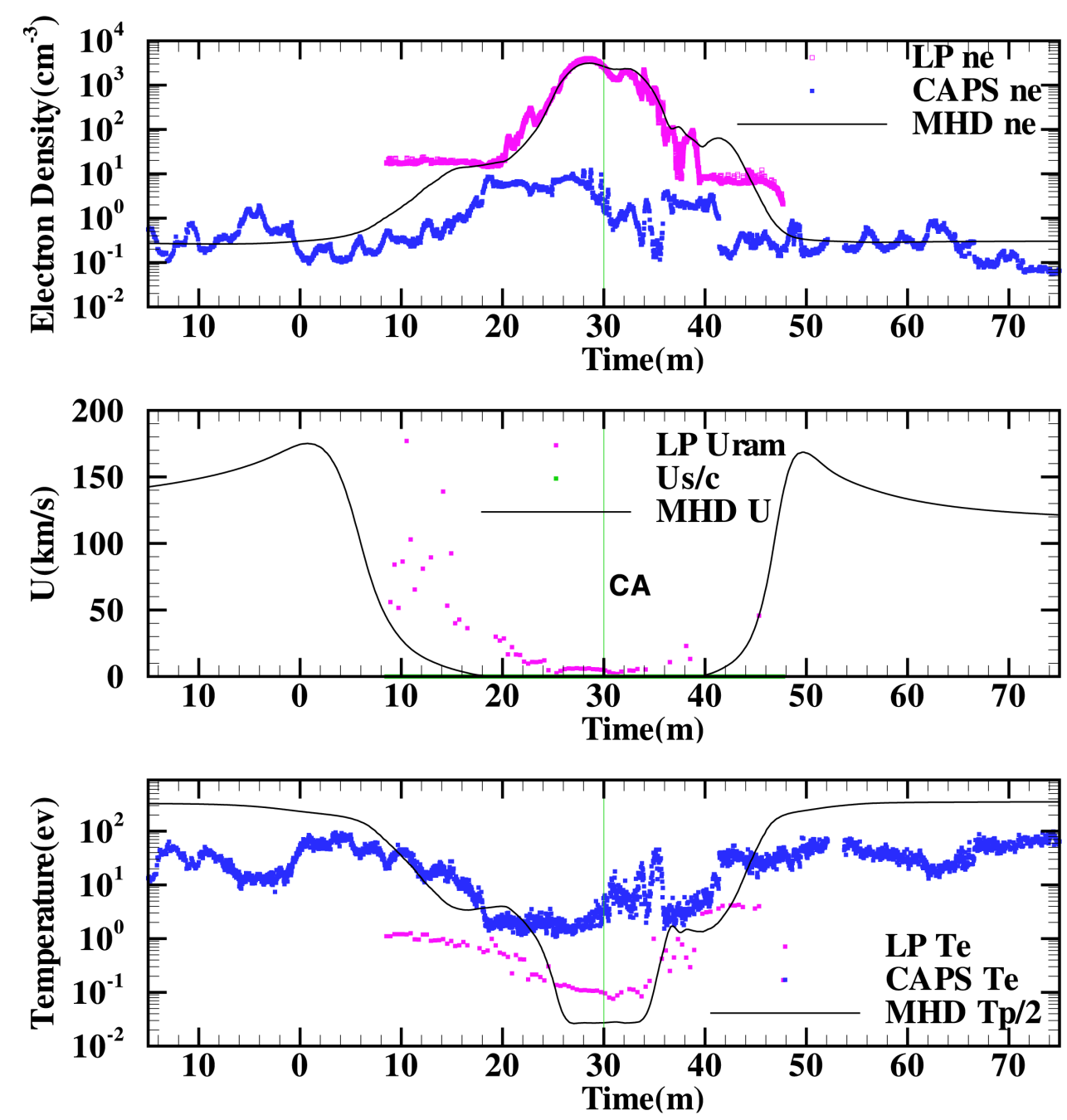

Figure 6. Calculated and measured values of electron density (upper), plasma velocity (middle), and temperature (lower) along the Cassini Ta trajectory near closest approach. The time in the $\mathrm{x}$-axis is relative to 1500 UT. The green lines correspond to the closest approach time of 1530 UT. The red point in the lower panel shows the ion temperature estimate from CAPS.

Langmuir probe electron temperatures are the indicator of the temperature of the bulk of the electrons in the ionosphere, while the CAPS makes the appropriate measurements outside that region. The ion temperature measured by CAPS is approximately $150 \mathrm{~K}$ around closest approach, consistent with neutral temperature value. Finally, it should be noted that the model calculates the plasma temperature, $\mathrm{Tp}$, which is the sum of the ion and electron temperatures. We plotted Tp/2, which implicitly assumes that electron and ion temperatures are equal, which is clearly a crude approximation at best and certainly not expected to be true in the ionosphere [cf. Roboz and Nagy, 1994].

[18] Finally, we plot in Figure 7 the calculated pressure profiles, magnetic field, and electron number density distribution along the ram direction for the measured $\mathrm{Ta}$ and Voyager upstream conditions, respectively. It is interesting to note the significant differences caused by the different upstream conditions and different solar zenith angles of the ram directions. This clearly demonstrates that previous model calculations that assumed Voyager upstream condition do not describe the situation found during the Ta flyby.

\subsection{Tb Flyby}

[19] The closest approach of the $\mathrm{Tb}$ flyby occurred on 13 December 2004, at 1138 UT. At that time Titan was located at about 10.5 hours Saturn local time (LT) similar to Titan's location during the Ta flyby, as shown in Figure 1. Like the Ta flyby, the observational data (from CAPS and $M A G)$ found significant variations in the surrounding plasma environments. CAPS data shows that the inbound electron number density ranged from $0.009 \mathrm{~cm}^{-3}$ to a value about 20 times larger between 0900 UT to 1100 UT, giving an average value of about $0.06 \mathrm{~cm}^{-3}$. The electron temperature values varied between $18.7 \mathrm{eV}$ and 10 times larger with a mean value of about $64.7 \mathrm{eV}$ (corresponding to $7.5 \times$ $\left.10^{5} \mathrm{~K}\right)$. CAPS data also show a significant difference between the inbound and outbound values in the electron density. The averaged outbound (from 1230 UT to 1430 UT) electron density increased significantly to $0.14 \mathrm{~cm}^{-3}$. The electron temperature varied from $7.3 \mathrm{eV}$ to $392 \mathrm{eV}$ with 

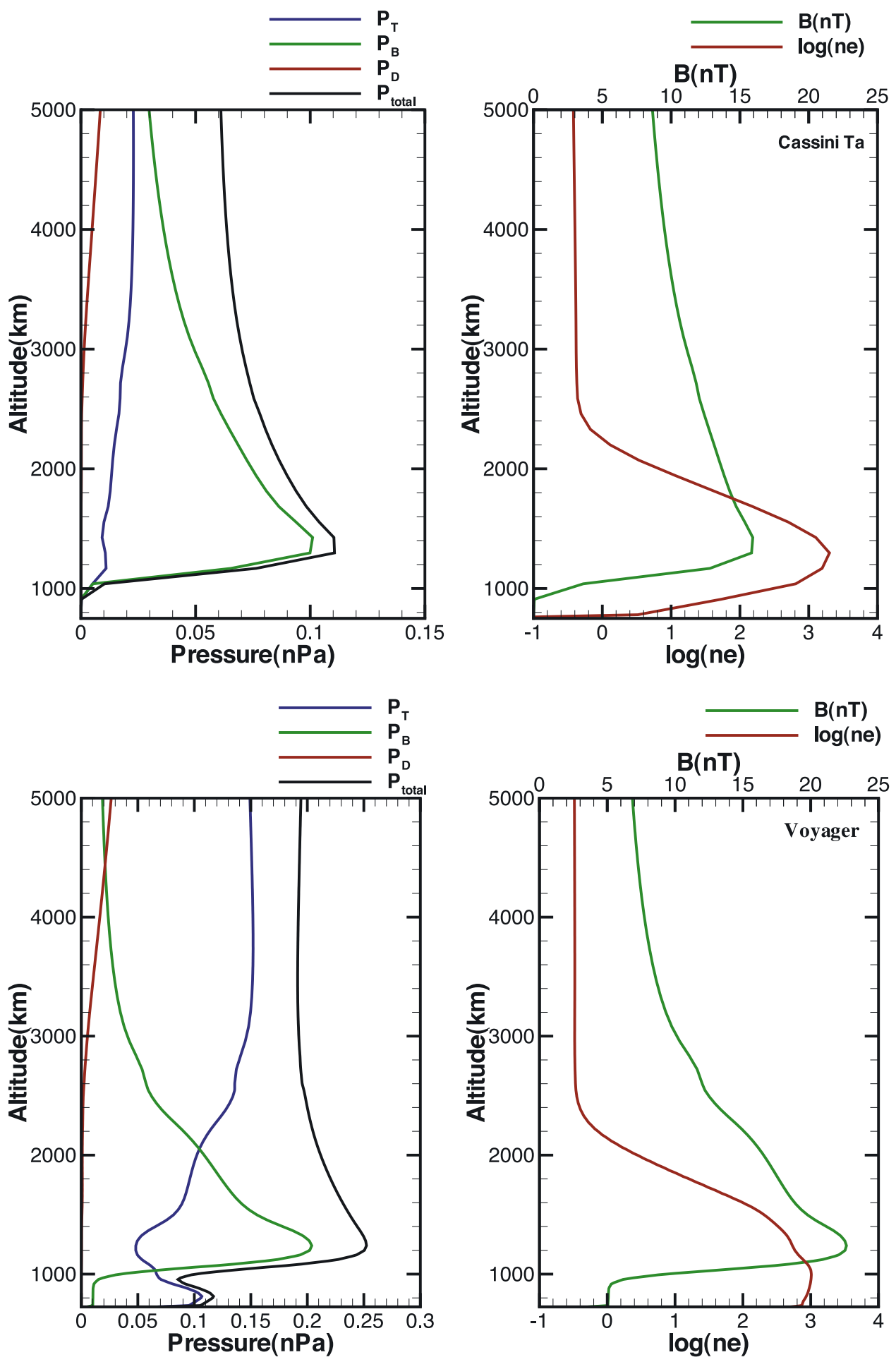

Figure 7. Model calculated pressure profiles; magnetic field, and electron density distributions along the ram direction for Ta (upper panel) and Voyager (lower panel) conditions.

the averaged electron temperature slightly larger than the inbound value. Compared with the observational data of Ta, the electron density is much smaller for the $\mathrm{Tb}$ flyby, especially during the inbound period, while the electron temperature is about twice of the corresponding value of Ta. The magnetic field data from the MAG team also show significant difference between the averaged inbound mag- netic field and the mean outbound magnetic field (see Table 2). The averaged value of the magnetic field magnitude of the Tb flyby is about $1 \sim 2 \mathrm{nT}$ smaller than that of the Ta flyby.

[20] Considering the significant variations in the upstream parameters, we selected the following values to simulate the Tb flyby (see Table 3). The upstream magnetic field was set 

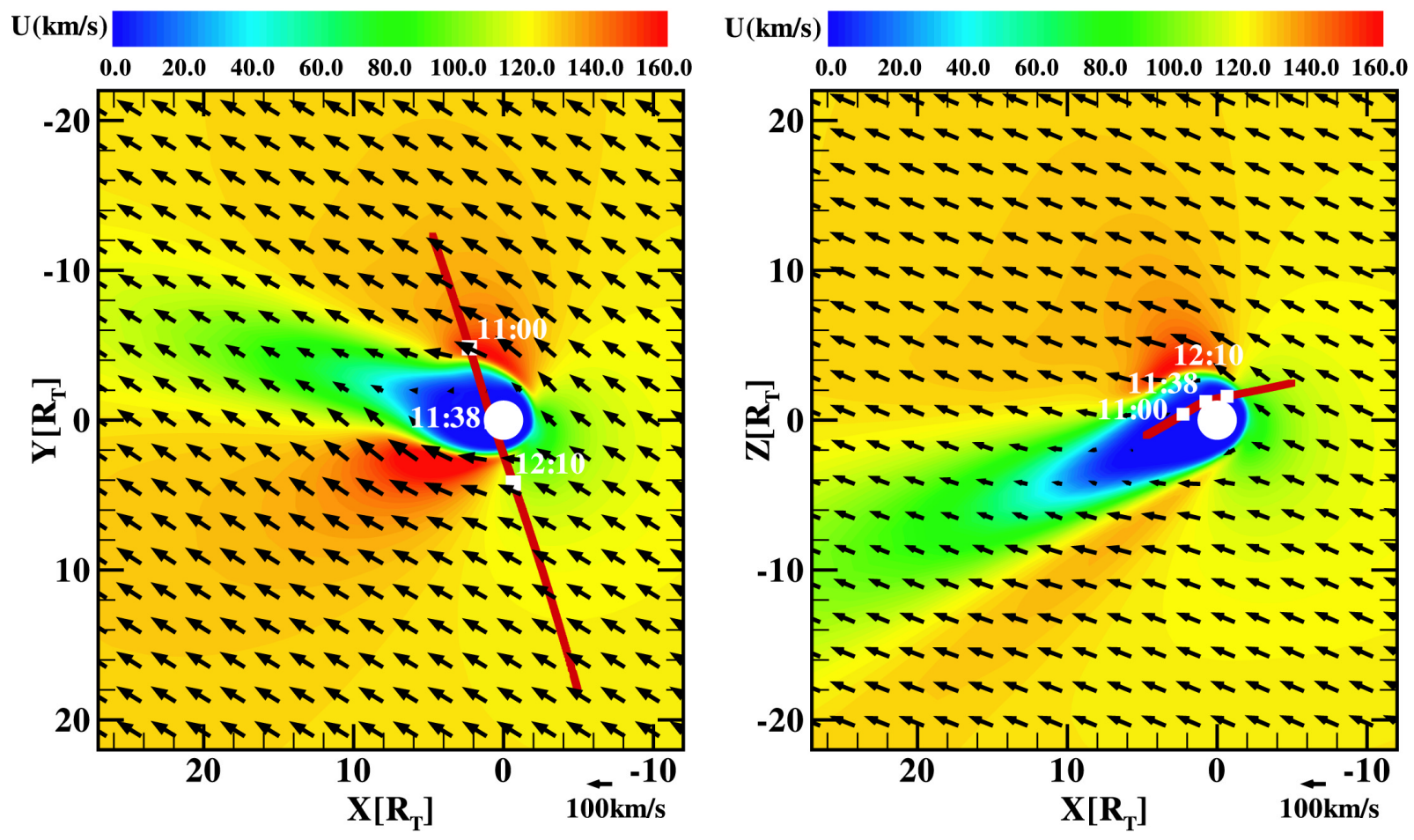

Figure 8. Calculated velocity values in $X-Y$ and $X-Z$ planes for Tb flyby. The color plots show the speed of the plasma, and the black arrows show the direction of the flow vector in the corresponding planes.

as $\mathbf{B}=(1.25,2.5,-3.5) \mathrm{nT}$. The upstream electron number densities were set to be $0.1 \mathrm{~cm}^{-3}$. Both the magnetic field and electron density are chosen as the averaged values of the inbound and outbound parameters. We assume the plasma ions are composed of $80 \% \mathrm{~L}^{+}$ions and $20 \%$ of $\mathrm{M}^{+}$ions. This ion composition corresponds to an average ion mass of $3.6 \mathrm{amu}$, which is smaller than what we selected for the Ta case, since during the whole Tb encounter, CAPS
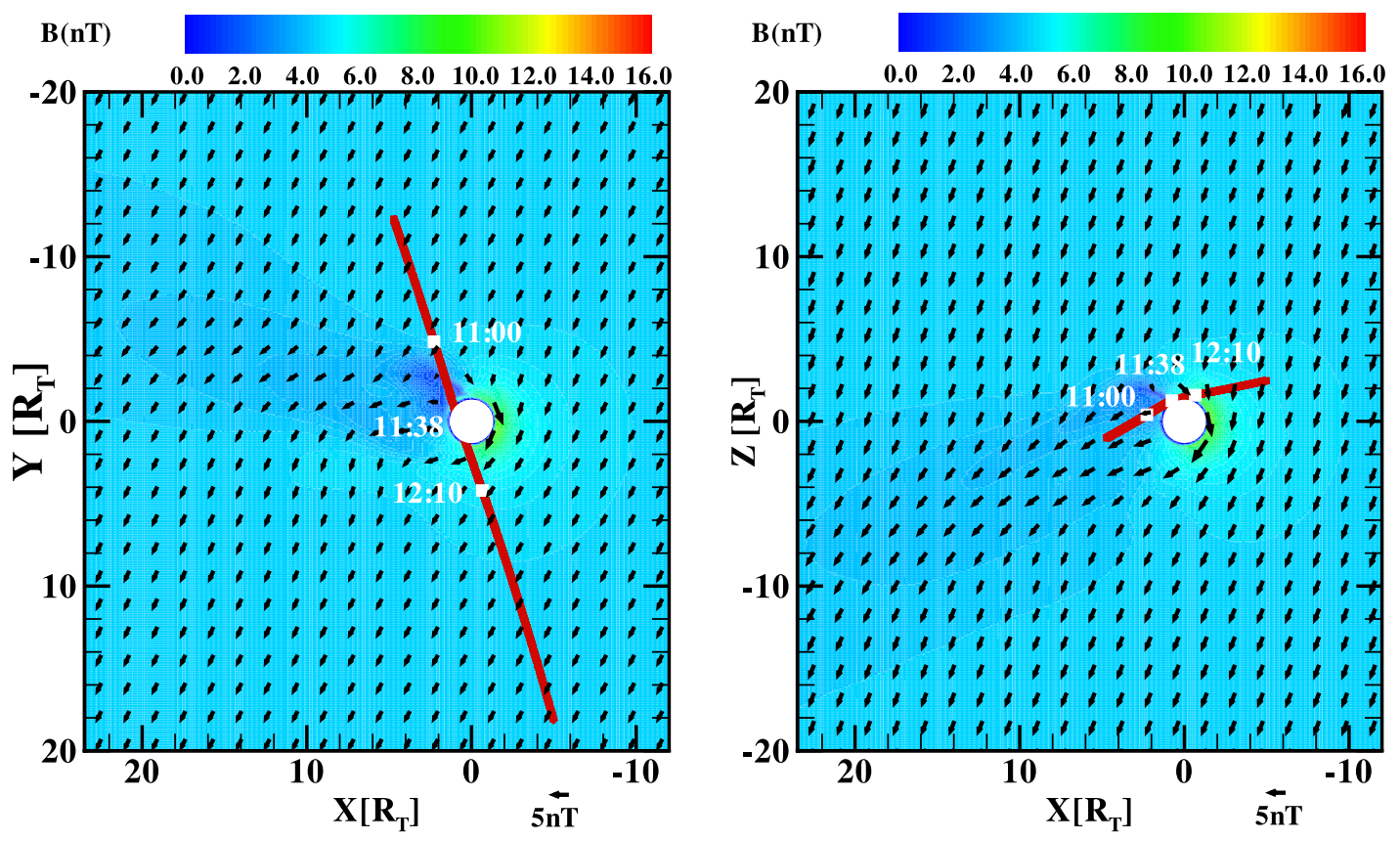

Figure 9. Calculated magnetic field values in $X-Y$ and $X-Z$ planes for Tb flyby. The color plots represent the strength of the magnetic field, and the black arrows indicate the direction of the magnetic field in the corresponding planes. 

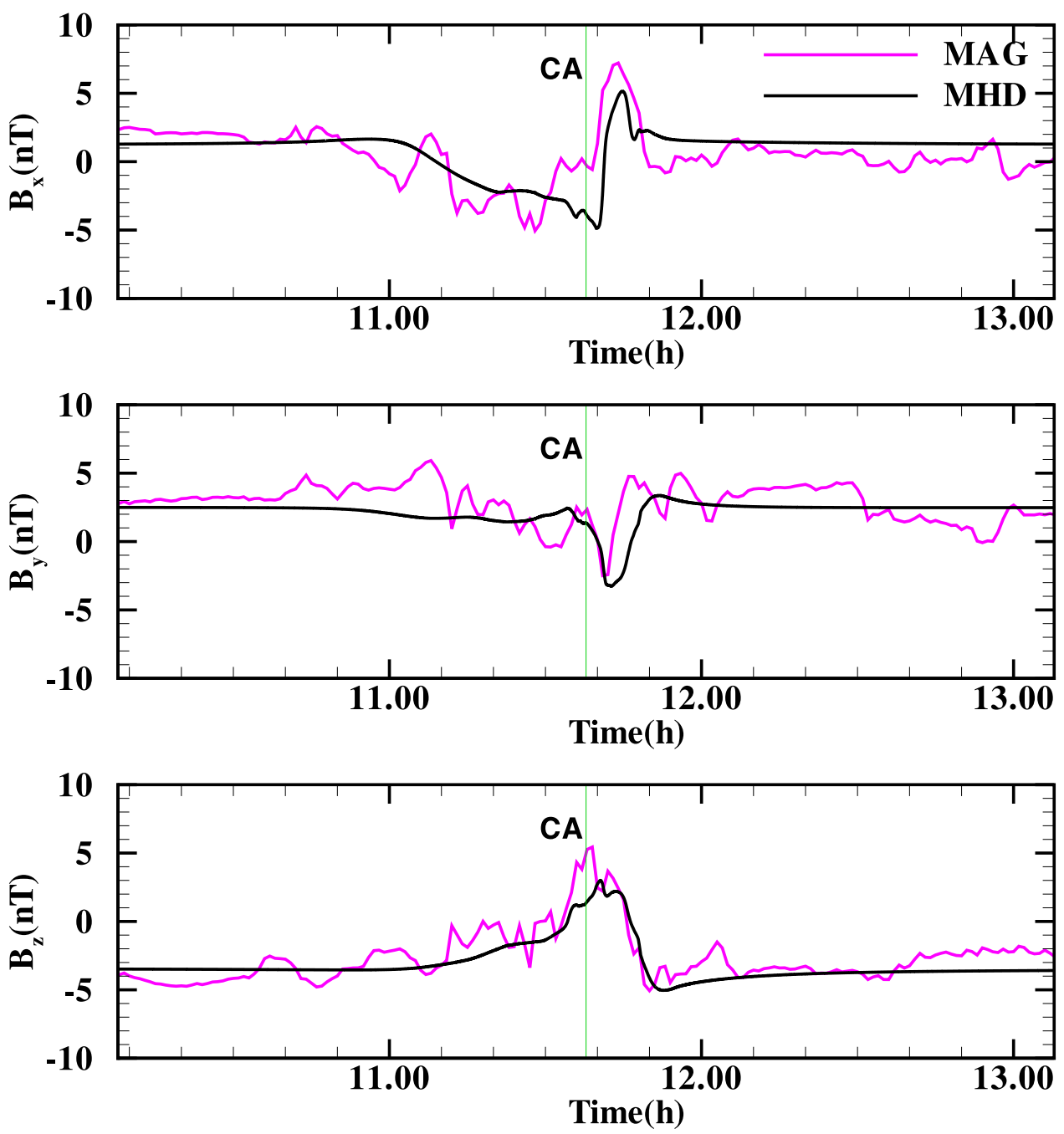

Figure 10. Calculated and measured magnetic field values along the Cassini Tb trajectory near closest approach. The green lines correspond to the closest approach time 1138 UT.

data indicated a smaller heavy ion content in the plasma flow relative to Ta [Szego et al., 2005]. The plasma temperature, $\mathrm{T}_{\mathrm{p}}$ was taken to be $750 \mathrm{eV}$. The value is about twice as high as the one used for Ta because the average electron temperature given by CAPS is about twice of the corresponding value of Ta. The plasma velocity was taken to be $\mathrm{u}=125 \mathrm{~km} \mathrm{~s}^{-1}$, with a $20^{\circ}$ northward component and $30^{\circ}$ anti-Saturn direction component. These parameters correspond to a subsonic $\left(\mathrm{M}_{\mathrm{S}}=0.68\right)$, super-Alfvenic $\left(\mathrm{M}_{\mathrm{A}}=0.77\right)$, subfastmagnetosonic $\left(\mathrm{M}_{\mathrm{f}}=0.62\right)$ flow, and a plasma $\beta=1.15$.

[21] Figure 8 shows the calculated velocities in the equatorial $(\mathrm{X}-\mathrm{Y})$ and vertical $(\mathrm{X}-\mathrm{Z})$ planes for the Tb flyby. The color plots show the speed of the plasma and the black arrows show the direction of the flow vector in the corresponding planes. The flow patterns in both the equatorial and vertical planes are similar to the case of Ta except the tail is tilted in different direction due to different upstream velocity direction. There is no bow shock formed in this case, just as was the case for Ta, the plasma flow is slower than the fast-magnetosonic speed.

[22] The calculated magnetic fields in the X-Y and X-Z planes for $\mathrm{Tb}$ flyby are shown in Figure 9. The color plots represent the magnitude of the total field and the black arrows show the direction of the magnetic field in the corresponding planes. The visible perturbed magnetic field region is smaller than that of Ta. Close to Titan, in both planes, the field lines are highly draped. The pile up of the magnetic field in front of Titan, due to the slowing down of the plasma flow, is not as strong as what we get for Ta case, since the upstream magnetic field is weaker and the upstream pressure is lower than the corresponding value of Ta. In both planes, a small current sheet is present in the wake region, similar to the case of Ta.

[23] Figure 10 shows the comparison between the calculated and observed magnetic field values along the $\mathrm{Tb}$ trajectory during the 3-hour interval centered on the closest approach (CA), shown by the green lines. The overall decreasing trend of $\mathrm{B}_{\mathrm{x}}$ between $1050 \mathrm{UT}$ and $1125 \mathrm{UT}$ shows the draping of the field lines in the north lobe, which is well reproduced by the model. The quick change of sign around1100 UT, where Cassini was about 4.3 RT from Titan, was likely caused by the fluctuation of the surrounding magnetic field and thus could not be reproduced by the 

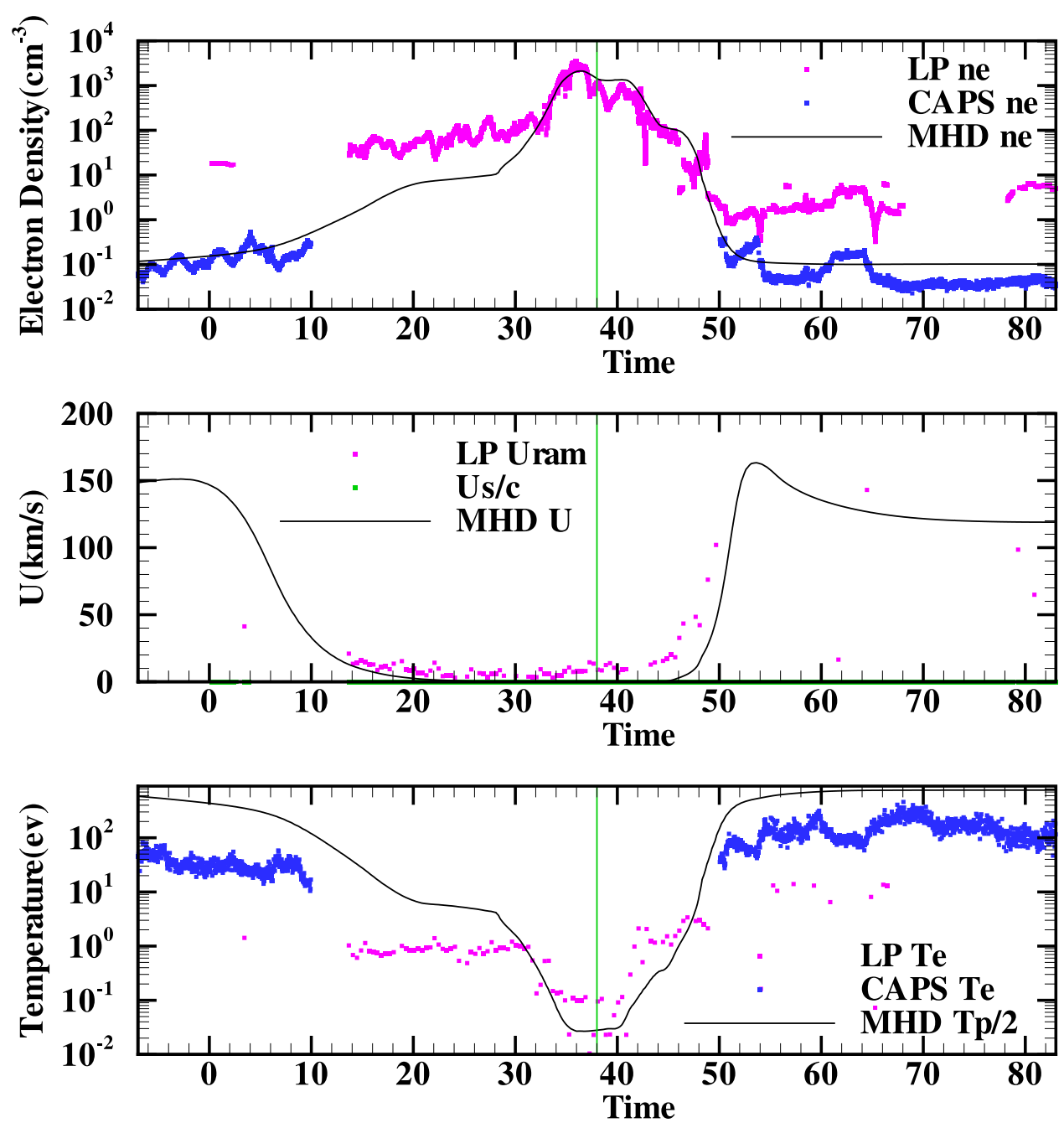

Figure 11. Calculated and measured values of electron density (upper), plasma velocity (middle), and temperature (lower) along the Cassini Tb trajectory near closest approach. The time in the $\mathrm{x}$-axis is relative to 1100 UT. The green lines correspond to the closest approach time 1138 UT.

model. The general changes of the $\mathrm{B}_{\mathrm{y}}$ and $\mathrm{B}_{\mathrm{z}}$ component are well reproduced by the model.

[24] Figure 11 shows the calculated and observed electron density (upper), plasma velocity (middle), and temperature (lower) along the Cassini $\mathrm{Tb}$ trajectory near closest approach. The time along the $\mathrm{X}$-axis is relative to $1100 \mathrm{UT}$. The vertical green lines indicate the closest approach time of 1138 UT. In the upper panel, the calculated electron densities agree reasonably well with the Langmuir probe data (between 1530 and 1545 UT) inside the ionosphere region and the CAPS data outside (1530-1545 UT), as was the case for Ta. The overall shape of the asymmetric distribution of the electron density can be explained by the photoionization and magnetospheric electron impact ionization. The inbound pass traversed the more sunlit dayside, whereas the outbound pass traversed the shaded region behind the atmospheric terminator. The electron densities from the Langmuir probe peaked two minutes before the CA with a maximum value of $3.2 \times 10^{3} \mathrm{~cm}^{-3}$ [Wahlund et al., 2005]. The model predicted that the peak density is at about the same time with a smaller value $2.1 \times$ $10^{3} \mathrm{~cm}^{-3}$. This discrepancy is likely to be due to the same reason as was discussed in the Ta section. In the middle panel, both the model results and the Langmuir probe data show a strong asymmetry of the plasma flow around CA. Our model results show that Cassini would have crossed the deceleration region at about $16 \mathrm{~min}$ before CA. This interval is longer than what we get for Ta. The slower drop of velocity for $\mathrm{Tb}$ might be connected with the slightly lower heavy ion content of the plasma during Tb [Szego et al., 2005]. Again, the observational data indicated a longer time period (about $36 \mathrm{~min}$ ) for the deceleration region before $\mathrm{CA}$ [Szego et al., 2005]. The measured electron temperatures

Table 4. Ion Escape Fluxes for Cassini Titan Flybys

\begin{tabular}{lccc}
\hline & Name & Escape Fluxes, $\mathrm{s}^{-1}, \mathrm{Ta}$ & Escape Fluxes, $\mathrm{s}^{-1}, \mathrm{~Tb}$ \\
\hline 1 & $\mathrm{~L}^{+}$ & $2.7 \times 10^{24}$ & $1.6 \times 10^{24}$ \\
2 & $\mathrm{M}^{+}$ & $1.0 \times 10^{24}$ & $4.4 \times 10^{23}$ \\
3 & $\mathrm{H}^{+}$ & $1.1 \times 10^{24}$ & $5.0 \times 10^{23}$ \\
4 & $\mathrm{H}^{+}$ & $1.4 \times 10^{23}$ & $8.6 \times 10^{22}$ \\
5 & $\mathrm{MHC}^{+}$ & $6.5 \times 10^{22}$ & $4.0 \times 10^{22}$ \\
6 & $\mathrm{HHC}^{+}$ & $1.7 \times 10^{22}$ & $1.5 \times 10^{22}$ \\
7 & $\mathrm{HNI}^{+}$ & $2.9 \times 10^{20}$ & $2.4 \times 10^{20}$ \\
& Total & $5.1 \times 10^{24}(104 \mathrm{~g} / \mathrm{s})$ & $2.6 \times 10^{24}(50.4 \mathrm{~g} / \mathrm{s})$ \\
\hline
\end{tabular}


near closest approach during the Tb flyby were lower (110 to $1200 \mathrm{~K}$ ) than those at Ta and thus appear to agree better with the model results although no ion temperature information is available.

[25] We calculated the ion escape fluxes from Titan, and they are shown in Table 4. The different upstream conditions and relative locations of Titan do result in different escape fluxes, as indicated in Table 4 . In both cases, the number escape flux of $\mathrm{L}^{+}$is the largest; however in terms of the mass escape fluxes, the $\mathrm{H}^{+}$ions represent the most important component. Although the calculated total escape fluxes are of the same order for both flybys, the one for Ta is about twice of that for $\mathrm{Tb}$. This might be related to the higher upstream dynamic pressure in the Ta case. These calculated fluxes are within the range of estimated values from both the Voyager [Gurnett et al., 1982] and early Cassini [Wahlund et al., 2005] observations. It should be noted that the escape fluxes presented here are from the ionosphere and from charge exchange and do not include escape caused by ion sputtering, which has been estimated to be a few times $10^{25} \mathrm{~s}^{-1}$ [e.g., Smith et al., 2004; Michael et al., 2005]. Finally, our estimated escape flux is comparable to the escape flux estimate of $9.3 \times 10^{24} \mathrm{~s}^{-1}$, resulting from photo and electron impact dissociation [Shematovich et al., 2003].

\section{Summary}

[26] Titan's interaction with Saturn's magnetosphere is an interesting and a very complex problem, in which Titan's location, solar illumination, and Saturn's magnetospheric plasma environment play important roles. In our model we have tried to include these factors to the degree that relevant information was available. We present results corresponding to the Ta and Tb flybys of Titan and compare the model results with the observed parameters. The overall comparison shows relatively good agreement. However, there are still some significant differences between the calculated and measured parameters. These differences may be caused by the uncertainties in the upstream conditions, such as ion temperature and velocity directions, and their possible temporal variations, as well as the limitations of the MHD model. Our model results have shown qualitatively, using some test runs, that the values along the trajectory are quite sensitive to the upstream conditions, which has been shown by the observations to be highly variable. To evaluate quantitatively the effects of changing upstream conditions, we will carry out time-dependent calculations in which we vary the upstream parameters. We have also begun changing our model to include thermal conductivity and the Hall effect. These are major changes the implementation of which will take some time to complete.

[27] Acknowledgments. The authors wish to acknowledge the many, many scientists, engineers, and support staff that made Cassini the success that it is. They are too many to list them individually, but they deserve a great deal of credit. The work done at the University of Michigan was supported by JPL contract 961176, NASA grant NAG5-13332, and NSF grant ATM0455729.

\section{References}

Acuña, M. H., et al. (1998), Magnetic field and plasma observations at Mars: Initial results of the Mars Global Surveyor Mission, Science, 279, 1676.
Backes, H., et al. (2005), Titan's magnetic field signature during the first Cassini encounter, Science, 308, 992.

Bauske, R., et al. (1998), A three-dimensional MHD study of solar wind mass loading processes at Venus: Effects of photoionization, electron impact ionization, and charge exchange, J. Geophys. Res., 103, 23,265. Brecht, S. H. (1997), Hybrid simulations of the magnetic topology of Mars, J. Geophys. Res., 102, 4743.

Brecht, S. H., J. G. Luhmann, and D. J. Larson (2000), Simulation of the Saturnian magnetospheric interaction with Titan, J. Geophys. Res., 105, 13,119

Cloutier, P. A., et al. (1999), Venus-like interaction of the solar wind with Mars, Geophys. Res. Lett., 26, 2685.

Crary, F. J., et al. (2006), Dynamics and composition of plasma at Titan, Science, in press.

Cravens, T. E., J. Vann, J. Clark, J. Yu, C. N. Keller, and C. Brull (2004), The ionosphere of Titan: An updated theoretical model, Adv. Space Res., 33, 212-215, doi:10.1016/J.asr.2003.02.012.

Cravens, T. E., et al. (2005), Titan's ionosphere: Model comparisons with Cassini Ta data, Geophys. Res. Lett., 32, L12108, doi:10.1029/ 2005GL023249.

Dougherty, M. K., et al. (2004), The Cassini Magnetic Field Investigation, Space Sci. Rev., 114, 331

Gan, L. C., N. Keller, and T. E. Cravens (1992), Electrons in the ionosphere of Titan, J. Geophys. Res., 97, 12,136.

Grard, R., A. Pedersen, S. Klimov, S. Savin, A. Skalsky, J. G. Trotignon, and C. Kennel (1989), First measurements of plasma waves near Mars, Nature, 341, 607

Gurnett, D. A., F. L. Scarf, and W. S. Kurth (1982), The structure of Titan's wake from plasma wave observations, J. Geophys. Res., 87, 1395

Hansen, K. C. (2001), MHD simulations of the magnetospheres of Jupiter and Saturn: Application to the Cassini mission, Ph.D. thesis, Univ. of Michigan, Ann Arbor, Mich

Harnett, E. M., and R. M. Winglee (2003), The influence of a mini-magnetopause on the magnetic pileup boundary of Mars, Geophys. Res. Lett. 30(20), 2074, doi:10.1029/2003GL017852.

Keller, C. N. (1992), One dimensional multispecies magnetohydrodynamic models of the ionosphere of Titan and the inner coma of comet P/Halley, Ph.D. thesis, Univ. of Kansas, Lawrence, Kan.

Ledvina, S. A., and T. E. Cravens (1998), A three-dimensional MHD model of plasma flow around Titan, Planet. Space Sci., 46, 1175

Ma, Y., A. F. Nagy, T. E. Cravens, I. V. Sokolov, J. Clark, and K. C. Hansen (2004a), Three-dimensional global MHD model prediction for the first close flyby of Titan by Cassini, Geophys. Res. Lett., 31, L22803, doi:10.1029/2004GL021215.

Ma, Y., A. F. Nagy, I. V. Sokolov, and K. C. Hansen (2004b), Threedimensional, multispecies, high spatial resolution MHD studies of the solar wind interaction with Mars, J. Geophys. Res., 109, A07211, doi:10.1029/2003JA010367.

Michael, M., R. E. Johnson, F. Leblanc, M. Liu, J. G. Luhmann, and V. I. Shematovich (2005), Ejection of nitrogen from Titan's atmosphere by magnetospheric ions and pick-up ions, Icarus, 175, 263.

Modolo, R., G. M. Chanteur, E. Dubinin, and A. P. Matthews (2005), Influence of the solar EUV flux on the Martian plasma environment, Ann. Geophys., 23, 433.

Murawski, K., and R. S. Steinolfson (1996), Numerical simulations of mass loading in the solar wind interaction with Venus, J. Geophys. Res., 101, 2547.

Nagy, A. F., and P. M. Banks (1970), Photoelectron fluxes in the ionosphere, J. Geophys. Res., 75, 6260.

Nagy, A. F., Y. Liu, K. C. Hansen, K. Kabin, T. I. Gombosi, M. R. Combi, D. L. DeZeeuw, K. G. Powell, and A. J. Kliore (2001), The interaction between the magnetosphere of Saturn and Titan's ionosphere, J. Geophys. Res., 106, 6151

Ogino, T., R. J. Walker, and M. G. Kivelson (1998), A global magnetohydrodynamic simulation of the Jovian magnetosphere, J. Geophys. Res., $103,225$.

Powell, K. G., P. L. Roe, T. J. Linde, T. I. Gombosi, and D. L. DeZeeuw (1999), A solution- adaptive upwind scheme for ideal magnetohydrodynamics, J. Comput. Phys., 154, 284.

Roboz, A., and A. F. Nagy (1994), The energetics of Titan's ionosphere, J. Geophys. Res., 99, 2087.

Schunk, R. W., and A. F. Nagy (2000), Ionospheres, Cambridge Univ. Press, New York

Shematovich, V. I., R. E. Johnson, M. Michael, and J. G. Luhmann (2003), Nitrogen loss from Titan, J. Geophys. Res., 108(E8), 5087, doi:10.1029/ 2003JE002094

Smith, H. T., R. E. Johnson, and V. I. Shematovich (2004), Titan's atomic and molecular nitrogen tori, Geophys. Res. Lett., 31, L16804, doi:10.1029/2004GL020580. 
Spreiter, J. R., A. L. Summers, and A. Y. Alksne (1966), Hydromagnetic flow around the magnetosphere, Planet. Space Sci., 14, 223.

Spreiter, J. R., A. L. Summers, and A. W. Rizzi (1970), Solar wind flow past nonmagnetic planets - Venus and Mars, Planet. Space Sci., 18, 1281.

Szego, K., et al. (2005), The global plasma environment of Titan as observed by Cassini Plasma Spectrometer during the first two close encounters with Titan, Geophys. Res. Lett., 32, L20S05, doi:10.1029/ 2005 GL022646.

Wahlund, J.-E., et al. (2005), Cassini measurements of cold plasma in the ionosphere of Titan, Science, 308, 986.

Waite, J. H., et al. (2005), Ion neutral mass spectrometer results from the first flyby of Titan, Science, 308, 982.
Young, D. T., et al. (2004), The Cassini Plasma Spectrometer, Space Sci. Rev., 114, 1

F. J. Crary, Southwest Research Institute, San Antonio, TX, USA.

T. E. Cravens, Department of Physics and Astronomy, University of Kansas, Lawrence, KS, USA.

A. J. Coates, Mullard Space Laboratory, University College London, London, UK.

M. K. Dougherty, Blackett Laboratory, Imperial College, London, UK.

K. C. Hansen, Y. Ma, A. F. Nagy, and I. V. Sokolov, Space Physics Research Laboratory, University of Michigan, Ann Arbor, MI, USA. (anagy@umich.edu)

J.-E. Wahlund, Swedish Institute of Space Physics, Uppsala, Sweden. 